\title{
Journal of Drug
}

\section{PEGylated Polylactide Micelles for Controlled Drug Delivery}

Jianxun Ding ${ }^{1 *}$ and Jixue Wang ${ }^{1,2}$

${ }^{1}$ Key Laboratory of Polymer Ecomaterials, Changchun Institute of Applied Chemistry, Chinese Academy of Sciences, Changchun 130022, P. R. China

${ }^{2}$ Department of Urology, the First Hospital of Jilin University, Changchun 130021, P. R. China

*Corresponding author: Dr. Jianxun Ding, Key Laboratory of Polymer Ecomaterials, Changchun Institute of Applied Chemistry, Chinese Academy of Sciences, 5625 Renmin Street, Changchun 130022, P. R. China; E mail: jxding@ciac.ac.cn

Article Type: Editorial, Submission Date: 31 August 2016, Accepted Date: 15 September 2016, Published Date: 13 February 2017.

Citation: Jianxun Ding and Jixue Wang (2017) PEGylated Polylactide Micelles for Controlled Drug Delivery. J. Drug 1(2): 14-16. doi: https://doi.org/10.24218/jod.2017.8.

Copyright: @ 2017 Jianxun Ding and Jixue Wang. This is an open-access article distributed under the terms of the Creative Commons Attribution License, which permits unrestricted use, distribution, and reproduction in any medium, provided the original author and source are credited.

Polylactide (PLA) is a type of widely used synthetic polymers with excellent biocompatibility and biodegradability [1]. Moreover, PLA with adjustable mechanical and physical properties, excellent workability, and low immunogenicity has been approved by the US Food and Drug Administration (FDA) for multiple medical applications clinically, such as drug delivery $[2,3]$, tissue engineering $[4,5]$, postoperative anti-adhesion $[6,7]$, medical device [8], and so on. PLA can be synthesized through the ring-opening polymerization (ROP) of lactide efficiently, which is catalyzed by a wide variety of organometallic catalysts [1]. As depicted in Figure 1, lactide acid is a chiral molecule existing in $\mathrm{L}$ and $\mathrm{D}$ isomers. As a result, PLA presents in three forms, that is, poly(L-lactic acid) (PLLA), poly(D-lactic acid)
(PDLA), and poly(D,L-lactic acid) (PDLLA) (Figure 1).

For drug delivery application, the hydrophobicity of PLA is a great restriction for systemically administered formulations [2]. The hydrophilic segments like poly (ethylene glycol) (PEG) are introduced to fabricate amphiphilic PLA-contained copolymers [9]. The PEG-PLA copolymers self-assemble into micelles with PEG shells and PLA cores. PLA cores serve as a reservoir of various hydrophobic drugs with high drug loading efficiency [10]. In the process of blood circulation in vivo, PEG endows micelles with "stealth" properties, that is, anti-protein adsorption and escape from reticuloendothelial system (RES), resulting extended circulation time $[11,12]$. In view of the above advantages,

L-lactic acid D-lactic acid<smiles>CC1OC(=O)C(C)OC1=O</smiles>

L-lactide<smiles>C[C@@H]1OC(=O)[C@@H](C)OC1=O</smiles>

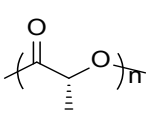
PDLA

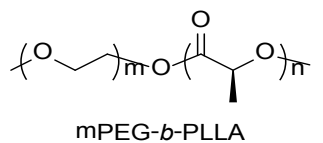<smiles>CCCCCCC(C)OC(=O)C(C)OCCOC</smiles>

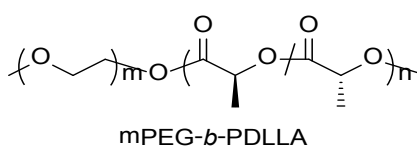<smiles>CC1OC(=O)C(C(C)C)OC1=O</smiles><smiles>CC(=O)C(C)OC(=O)C(C)Oc1ccccc1</smiles>

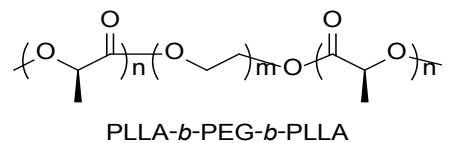

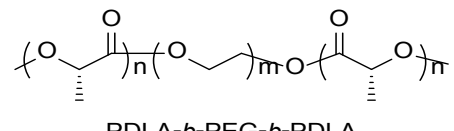

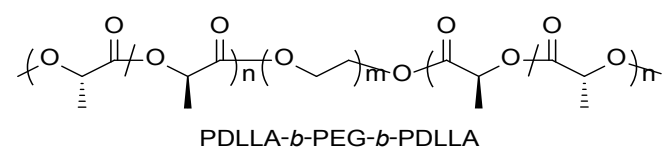

Figure 1: Chemical structures of L-lactic acid, D-lactic acid, L-lactide, D-lactide, D,L-lactide, PLLA, PDLA, PDLLA, mPEG- $b$-PLLA, mPEG- $b$-PDLA, mPEG- $b$-PDLLA, PLLA- $b$-PEG- $b$-PLLA, PDLA- $b$-PEG- $b$-PDLA, and PDLLA- $b$-PEG- $b$-PDLLA 
PEG-PLA micelles has been widely researched and applied as promising nanocarriers for controlled drug delivery $[2,9,13]$. Most encouragingly, Genexol ${ }^{\circledR}$-PM, a paclitaxel (PTX)-loaded PEG-PDLLA micelle, is the only clinically approved nanoscale polymer chemotherapeutic up to now, which was exploited by Samyang Genex Co. (Seoul, Korea) [14,15].

With the development of PEG-PLA micelles as drug delivery platforms, the poor stability of micelles in blood circulation limits their improvement in efficacy. As a benefit of different configurations of PLA, that is, PLLA and PDLA, the PEGPLLA/PEG-PDLA stereocomplex micelles (SCMs) exhibit both upregulated thermodynamic and kinetic stability (Figure 2).

As depicted in Figure 2, several PLA SCMs have been developed tocontrollably deliver different antitumor drugs, such as doxorubicin (DOX) and 10-hydroxycamptothecin (HCPT), in our research group $[2,9,10,13]$. As a typical example, the DOX-loaded PLLA-based micelle (PLM/DOX), PDLA-based micelle (PDM/DOX), and SCM (SCM/DOX) were fabricated [9]. Compared with PDM/DOX and PLM/DOX, SCM/DOX exhibited the smallest hydrodynamic diameter $\left(D_{h}\right)$, the most effective cell endocytosis, and the strongest antineoplastic efficacy in vitro. Moreover, the cholesterol-enhanced DOX-loaded PLLA, PDLA, and PLLA/PDLA-participated micelles, that is, CPLM/ DOX, CPDM/DOX, and CSCM/DOX, were further prepared for controlled drug delivery [13]. Similarly, compared with CPLM/
DOX and CPDM/DOX, CSCM/DOX showed the smallest $D_{\mathrm{h}}$ and the slowest DOX release. More importantly, all the DOXloaded micelles, especially SCM/DOX and CSCM/DOX, exhibited the excellent antiproliferative efficacy that was equal to or even better than free DOX $[9,13]$. The first modified SCM for optimized drug delivery was prepared in our research group [13].

Although PEG-PLA micelles have made great progress in the field of drug delivery, the following aspects are worth to be studied further.

- The sizes can be adjusted by changing the composition ratio and lengths of PEG and PLA blocks. Appropriate size will optimize the enhanced permeability and retention (EPR) effect and tumor tissue penetration in vivo.

- The surface can be modified to adjust the surface potential and increase biological function. The modification can make the micelles better adapt to the needs of different drug delivery systems.

- The mesomeric PLA micelles and PLLA/PDLA SCMs exhibit superior drug-loading performance and stability, and more controlled drug release. These two types of micelles are worthy of further preclinical and clinical researches.

Through continuous improvement of size, stability, circulation, intratumoral accumulation, tumor penetration, and intracellular

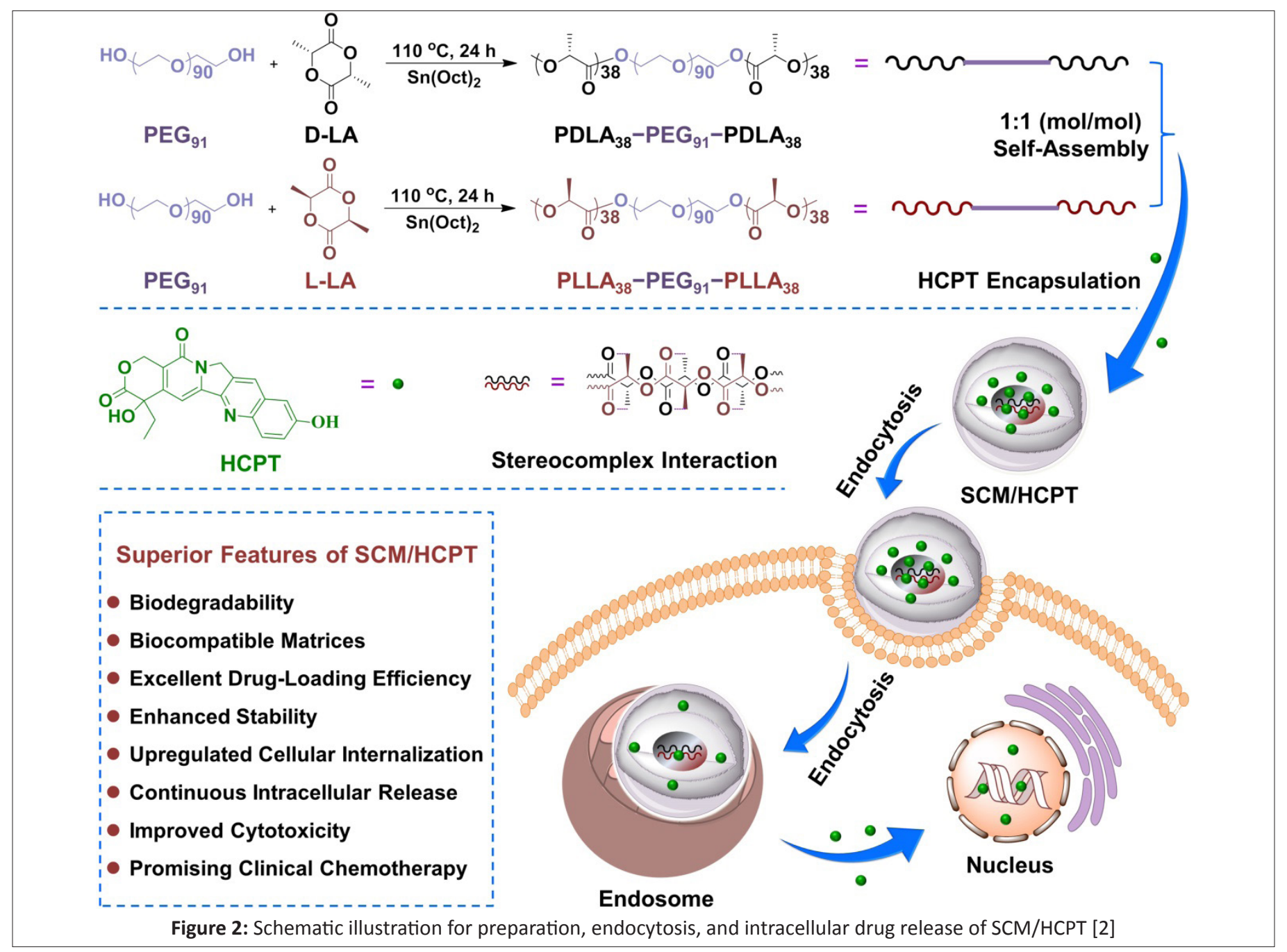


Citation: Jianxun Ding and Jixue Wang (2017) PEGylated Polylactide Micelles for Controlled Drug Delivery. J. Drug 1(2): 14-16. doi: https://doi. org/10.24218/jod.2017.8.

drug release, the time of PEG-PLA micelles for the benefit of cancer patients is not far off.

\section{Acknowledgements}

The editorial was financially supported by the National Natural Science Foundation of China (Nos. 51303174 and 51673190).

\section{References}

1. Pang XA, Zhuang XL, Tang ZH, Chen XS. Polylactic acid (PLA): Research, development and industrialization. Biotechnol J. 2010; 5(11):1125-36. doi: 10.1002/biot.201000135.

2. Feng C, Piao M, Li D. Stereocomplex-Reinforced PEGylated Polylactide Micelle for Optimized Drug Delivery. Polymers.2016; 8:165. doi: 10.3390/polym 8040165.

3. Ding J, Chen L, Xiao C, Chen L, Zhuang X, Chen X. Noncovalent interaction-assisted polymeric micelles for controlled drug delivery. Chem Commun. 2014; 50:11274-90. doi: 10.1039/C4CC03153A.

4. Zhang ZZ, Jiang D, Wang SJ, Qi YS, Ding JX, Yu JK, et al. Scaffolds drive meniscus tissue engineering. RSC Adv. 2015; 5:77851-9. doi: 10.1039/C5RA13859K.

5. Zhang J, Yang SG, Ding JX, Li ZM. Tailor-made poly (L-lactide)/poly (lactide-co-glycolide)/hydroxyapatite composite scaffolds prepared via high-pressure compression molding/salt leaching. RSC Adv. 2016; 6:47418-26. doi: 10.1039/C6RA06906A.

6. Song Z, Shi B, Ding J, Zhuang X, Zhang X, Fu C, et al. A comparative study of preventing postoperative tendon adhesion using electrospun polyester membranes with different degradation kinetics. Sci China Chem. 2015; 58:1159-68. doi: 10.1007/s11426-015-5425-7.

7. Shi B, Ding J, Wei J, Fu C, Zhuang X, Chen X. Drug-Incorporated Electrospun Fibers Efficiently Prevent Postoperative Adhesion. Curr Pharm Des. 2015; 21(15):1960-6. doi: 10.2174/138161282166615 0302152420.
8. Bergström JS, Hayman D. An Overview of Mechanical Properties and Material Modeling of Polylactide (PLA) for Medical Applications. Ann Biomed Eng. 2016; 44(2):330-40. doi: 10.1007/s10439-0151455-8.

9. Wang J, Shen K, Xu W, Ding J, Wang X, Liu T, et al. Stereocomplex micelle from nonlinear enantiomeric copolymers efficiently transports antineoplastic drug. Nanoscale Res Lett. 2015; 10(1):907. doi: 10.1186/s11671-015-0907-2.

10. Liu DH, Ding JX, Xu WG, Song XF, ZhuangXL, Chen XS. Stereocomplex Micelles Based on 4-Armed Poly (ethylene glycol)-Polylactide Enantiomeric Copolymers for Drug Delivery. Acta Polym Sin. 2014:1265-73. doi: 10.11777/j.issn1000-3304.2014.14007.

11. Chen J, Ding J, Xiao C, Zhuang X, Chen X. Emerging antitumor applications of extracellularly reengineered polymeric nanocarriers. Biomater Sci. 2015; 3(7):988-1001. doi: 10.1039/c5bm00044k.

12. Nekkanti V, Rueda J. Nanoparticles for Improved Delivery of Poorly Soluble Drugs. J. Drug. 2016; 1(1):18-27.

13. Wang J, Xu W, Ding J, Lu S, Wang X, Wang C, et al. CholesterolEnhanced Polylactide-Based Stereocomplex Micelle for Effective Delivery of Doxorubicin. Materials.2015; 8:216-30. doi: 10.3390/ ma8010216.

14. Lee JL, Ahn JH, Park SH, Lim HY, Kwon JH, Ahn S, et al. Phase II study of a cremophor-free, polymeric micelle formulation of paclitaxel for patients with advanced urothelial cancer previously treated with gemcitabine and platinum. Invest New Drugs. 2012; 30(5):1984-90. doi: 10.1007/s10637-011-9757-7.

15. Ahn HK, Jung M, Sym SJ, Shin DB, Kang SM, Kyung SY, et al. A phase II trial of Cremorphor EL-free paclitaxel (Genexol-PM) and gemcitabine in patients with advanced non-small cell lung cancer. Cancer Chemother Pharmacol. 2014; 74(2):277-82. doi: 10.1007/ s00280-014-2498-5. 